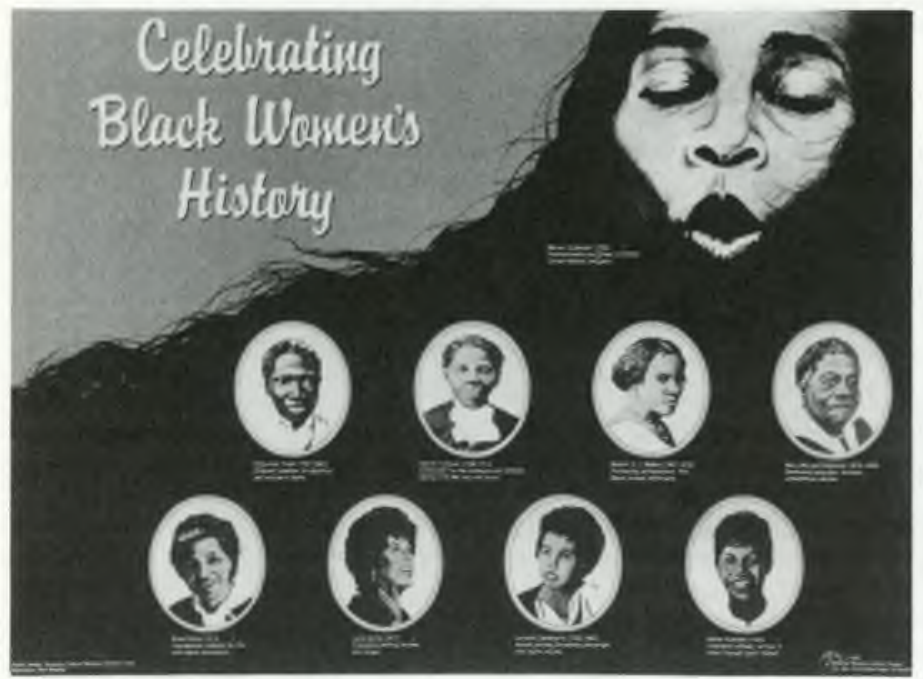

February is Black History Month and this 3-color poster celebrates black women's history by honoring nine prominent African American women: Marian Anderson, Sojourner Truth, Harriet Tubman, Madam C. J. Walker, Mary McLeodBethune, Rosa Parks, Lena Horne, Lorraine Hansberry, and wilma Rudolph. The poster is available for $\$ 6.00$ (plus $\$ 3.50$ for shipping $\&$ handling) from the National Women's History Project, 7738 Bell Road, Windsor, CA 95492.

\title{
Government Printing Office expands database access
}

In June of 1994, the Government Printing Office (GPO) unveiled GPO Access, an online system of databases which is the result of the GPO Electronic Information Access Enhancement Act of 1993. In its original form, GPO Access offered a single subscription to every federal depository library of each of the following databases: Congressional Record and Index, Federal Register and Index, and Enrolled Bills of the 103rd Congress.

In September of 1994, GPO announced a new program to enhance the accessibility of these databases through GPO Access Gateways, which originally included six university libraries and one public library. This program was expanded in October of 1994 to include any library willing to sign on and support a Gateway. Unlike GPO Access as it was originally proposed, which supplied access to the databases for only one user from one IP address, the Gateways will support multiple users simultaneously from one site. In addition to expanding the availability of GPO Access, GPO has also announced that the number of databases will expand. The United States Code has recently been added, as has the database that will contain the bills of the 104th Congress. GPO hopes to add the Code of Federal Regulations and the Government Information Locator in the near future.
Here are the GPO Access Gateways currently in operation and how to access them:

COIN: Telnet 128.206.1.3 or dial (314) 8847000; login as GUEST; Main Menu select "Government Center"; select GPO ACCESS.

Seattle Public Library: Telnet 198.137. 188.2 or dial (206) 386-4140; login as library (lower case); select VT100; select Internet twice; from Gateways menu select GPO Access.

Georgia Southern University: Telnet gsvms2.cc.gasou.edu or dial into GSnet (9600 baud) at (912) 681-0005; modem settings are 8 data bits, no parity, 1 stop bit; terminal emulation VT100; press < Enter > twice; from GSnet prompt enter: connect gsvms2; login as Info; select Government Printing Office Access; users with slower modems may dial into PeachNet at (912) 681-0500; at the PeachNet prompt enter: connect gsvms2.cc.gasou.edu.

Penn State University: Telnet lias.psu.edu or dial (814) 865-5427; communication settings are full duplex, no parity, 8 bits, 1 stop bit; VT100 emulation is recommended. For dial access: when connection is established press $<$ Enter $>$ once. For dial access and Internet access: respond to prompts for terminal emulation and user ID (press < Enter>); at LIAS screen type: Select; from Select menu choose GPO Access; to exit LIAS, type exit.-Maurie Caitlin Kelly, University of Illinois at Chicago 